\title{
Latest emulsion detector for cosmic ray observation: highly sensitive emulsion film and high-speed readout system
}

\author{
Hiroki Rokujo* \\ Nagoya University \\ E-mail: rokujodflab.phys.nagoya-u.ac.jp \\ for the GRAINE collaboration \\ Aichi University of education, ISAS/JAXA, Kobe University, Nagoya University, Okayama \\ University of science, Utsunomiya University
}

\begin{abstract}
A nuclear emulsion is used in a high-resolution 3D tracking device. AgBr crystals of $0.2 \mu \mathrm{m}$ size that are penetrated by a charged particle grow, via a chemical development process, into 0.8 $\mu \mathrm{m}$ silver grains that can be observed as a track by using a microscope. Recent fully automated readout systems have enabled not only high-resolution measurements but also large-scale experiments (accelerator and balloon-borne experiments, cosmic ray muon radiography, etc.). Since 2010, properties of the emulsion detector have drastically changed. We have introduced a system of nuclear emulsion gel production to the laboratory in Nagoya University, Japan, and have started self-development and supply of the new gel, instead of relying on photographic film companies. We have also developed a next-generation readout system, the Hyper Track Selector (HTS). The scanning speed is designed to be $0.9 \mathrm{~m}^{2} / \mathrm{h}$ (100 times faster than the current system). GammaRay Astro-Imager with Nuclear Emulsion (GRAINE) is a cosmic gamma- ray observation project that uses a balloon-borne emulsion detector. The angular resolution of the emulsion gamma-ray telescope $\left(0.08^{\circ} @ 1-2 \mathrm{GeV}\right)$ is one order of magnitude higher than that of the Fermi-LAT. In addition, it has polarization sensitivity using the pair creation mode. A search for exotic particles and measurements of short-lived particle production rates in cosmic rays at balloon altitudes will be also conducted. In the second GRAINE balloon-borne experiment in 2015 May, $50 \mathrm{~m}^{2}$ of the new highly sensitive emulsion films were used for the medium-scale gamma-ray telescope (aperture area $3600 \mathrm{~cm}^{2}$ ), with which we demonstrate the imaging performance. We present the status of the latest emulsion detector and readout system based on results of performance tests conducted for the GRAINE balloon-borne experiment in 2015.
\end{abstract}

The 34th International Cosmic Ray Conference,

30 July- 6 August, 2015

The Hague, The Netherlands

${ }^{*}$ Speaker. 


\section{Introduction}

Nuclear emulsion is used as a 3D high-resolution tracking detector. Generally, nuclear emulsion gel is made of $\sim 0.2 \mu \mathrm{m}$ silver bromide $(\mathrm{AgBr})$ crystals dispersed in gelatin. The emulsion plate, which is made by coating one or both sides of a plastic base with emulsion gel, records trajectories of charged particles that penetrate layers of the emulsion gel. After chemical development processes, the crystals grow into $\sim 0.8 \mu \mathrm{m}$ silver grains. We can observe a line of grains as a track by using a microscope. The intrinsic position resolution of a nuclear emulsion is $200 / \sqrt{12}=58$ $\mathrm{nm}$.

So far, many discoveries in the particle physics field have occurred during experiments using this nuclear emulsion technique. In 1946, Powell and Ilford developed an emulsion that was more sensitive than the normal half-tone emulsion by increasing the $\mathrm{AgBr}$ content in the gelatin. This led to remarkable progress in the recognition of particle tracks and to the discovery of charged pions in emulsion plates exposed to cosmic-rays [四]. Niu installed Emulsion Cloud Chambers, which consist of multiple sandwiches of nuclear emulsion plates and metal plates, on an aircraft and studied high-energy interactions of cosmic rays. In 1971, his group discovered an event containing a charmed particle before the descovery of J/ $\psi$ discovery [2]. Since the 1980's, automated emulsion scanning systems have been continuously developed [3] [团][圆][园]. This has made the task of analysis, the "bottleneck" part of emulsion experiments, efficient and contributed to the discovery of the tau neutrino $[\nabla]$, detection of double hyper nuclei [[]], and observation of $v_{\mu} \rightarrow v_{\tau}$ oscillation in appearance mode [0].

Since 2010, the following two topics drastically have drastically changed the circumstances of using an emulsion detector: highly sensitive nuclear emulsions and high-speed scanning systems. In this paper, we report about these latest circumstances with regard to emulsion detectors and their application with respect to a recent cosmic ray experiment.

\section{Latest emulsion detector technology}

\subsection{Highly sensitive nuclear emulsion gel}

Nuclear emulsions have been developed and produced by Ilford, Kodak and Fuji Film. However, owing to rapid recent digitalization in the photographic industry, departments dedicated to the development of nuclear emulsions have been closed down. Under these circumstances, in 2010, an emulsion group at Nagoya university introduced a gel production machine to the laboratory, and started developing nuclear emulsion in cooperation with former members of Fuji Film.

The formation of $\mathrm{AgBr}$ crystals, the main component of nuclear emulsion production, is performed by adding $\mathrm{Ag}^{+}$and $\mathrm{Br}^{-}$to a gelatin solution with precise control of addition speed, mixing speed, temperature, and so on. Physicists started self-development and supply of the new gel by controlling the size of a crystal or using chemical additives. Naganawa and former members of Fuji Film worked on sensitivity improvement of nuclear emulsion [ए]]. They increased the $\mathrm{AgBr}$ content in the gelatin from the typical $30-45 \%$ to 55\% in volume, as in Powell's attempt. Figure W shows microscopic views of a typical emulsion film and a highly sensitive emulsion film exposed to an electron beam horizontally. The grain density of the highly sensitive film is two times greater than that of the typical film with equal fog density. 


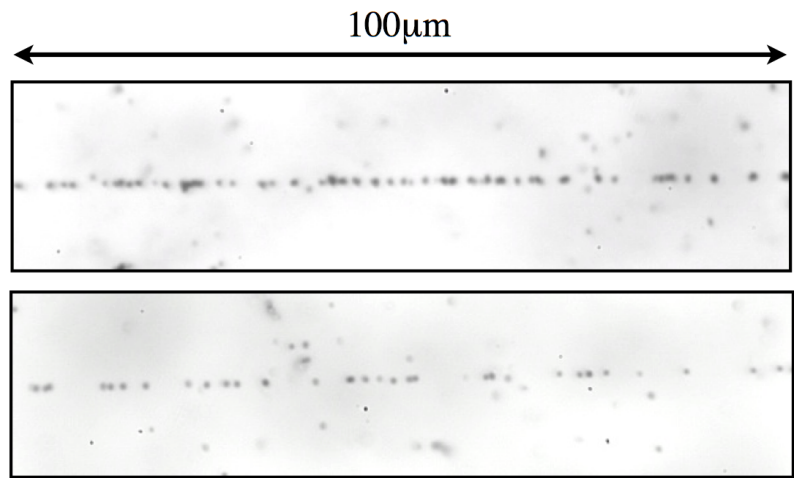

Figure 1: Microscopic view of highly sensitive film (upper) and typical film (lower)

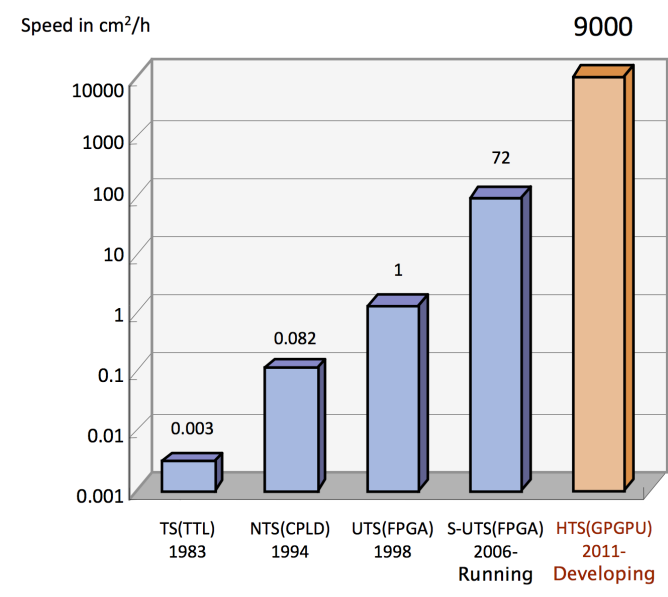

Figure 2: Evolution of scanning speed of emulsion film readout systems

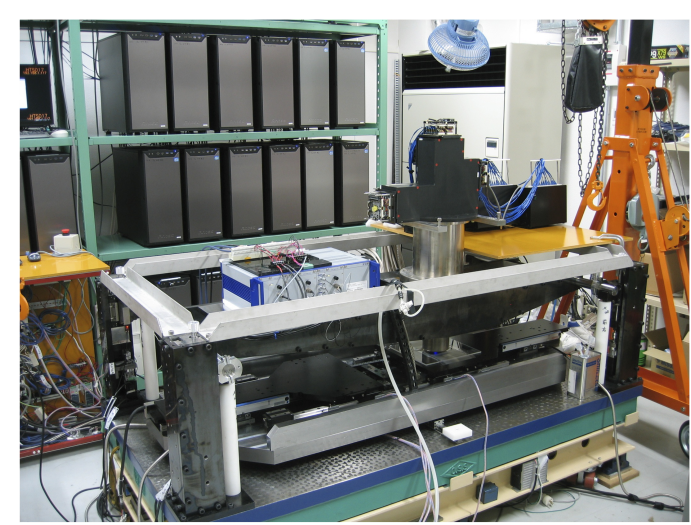

Figure 3: Picture of HTS

\subsection{High-speed readout system}

The development of a high-speed readout system, the Hyper Track Selector (HTS), is ongoing at Nagoya university. Figure $\square$ shows scanning speeds of track selectors. Their performances continue to increase with each passing era. The designed scanning speed of the HTS is 9000 $\mathrm{cm}^{2} / \mathrm{h}$, which is 100 times faster than that of the current-generation S-UTS.

Figure $[$ is a picture of the HTS. The key to increasing speed is a huge objective lens. It realizes a $5 \mathrm{~mm} \times 5 \mathrm{~mm}$ wide field of view (FOV). In addition to the objective lens, the HTS consists of 72 CMOS image sensors (each $2 \mathrm{M}$ pixels, $340 \mathrm{fps}$ ) that cover the FOV, a piezo-driven Z-axis stage that changes the depth of the focal plane within an emulsion layer, a motor-driven $X Y$ axes stage that moves to the next view precisely and quickly after capturing images of the current view, and 36 PCs mounting GPGPUs for calculation of track recognition in an emulsion layer.

Currently, actual scanning speed is $2500 \mathrm{~cm}^{2} / \mathrm{h}$, and the HTS will be fine-tuned steadily toward the designed performance. 


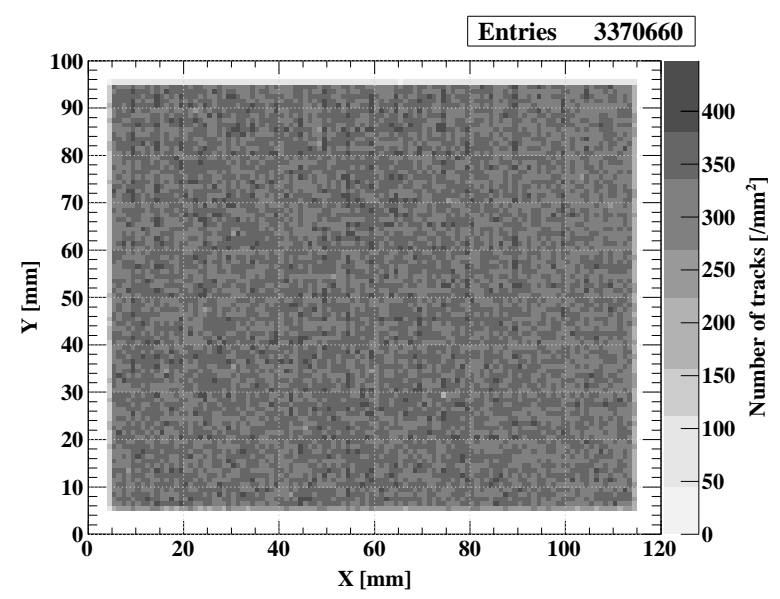

Figure 4: Position distribution of scanned track data

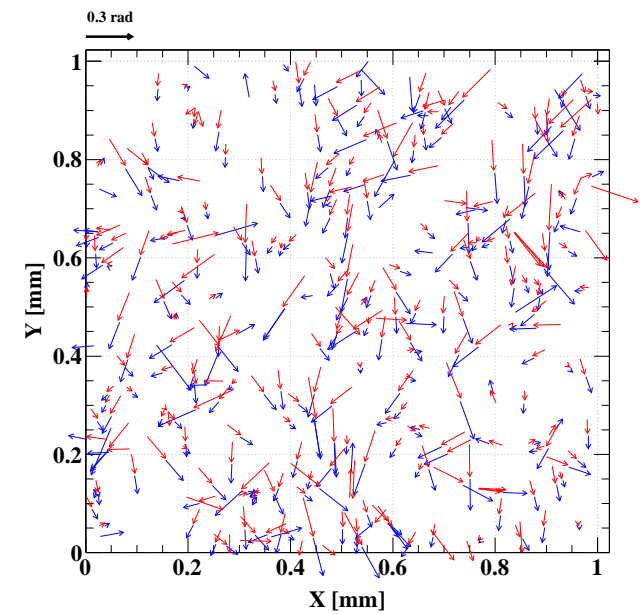

Figure 5: Enlarged view of scanned track data with arrow expression

\section{Muon beam test}

Here, we show data acquired by highly sensitive nuclear emulsion film and the HTS.

Films were produced by pouring melted gel on the both sides of a $180 \mu \mathrm{m}$-thick polystyrene base. The thickness of each emulsion layer was $70 \mu \mathrm{m}$. This was cut into two films, each 125 $\mathrm{mm} \times 100 \mathrm{~mm}$, and both were vacuum-packed with laminated shading films. The chamber was set at the muon pit for the T2K neutrino oscillation experiment in J-PARC [W]. A test beam was shot toward the neutrino beam line. After one shot exposure $\left(2.45 \times 10^{11}\right.$ protons-on-target $)$, the chamber was recovered and developed at Nagoya university.

Using the HTS, $110 \mathrm{~mm} \times 90 \mathrm{~mm}$ areas of these films were scanned. Figure $\$$ shows the number of recognized tracks in a highly sensitive emulsion film. Several hundreds tracks were read out in a $1 \mathrm{~mm} \times 1 \mathrm{~mm}$ area. Figure $\$$ displays an enlarged view of one square millimeter area. Each arrow indicates track information. The starting position, direction, and length of arrow correspond to position, 3D vector and angle for the incident track, respectively. The thick arrow at the upper left indicates a 0.3 radian incident angle relative to the normal vector of the film surface. Red and blue arrows show track information recorded in a up-stream film and a down-stream film, respectively, in a packed chamber.

These track data are almost-raw data outputted from the HTS. It is easy to discriminate combinations of penetrating tracks that should be reconstructed with no specific selection cut. The percentage of background noise or false signal is very low. It was confirmed that highly sensitive emulsion film and the HTS provide high-quality track data.

\section{Application in cosmic ray experiment}

Currently, a next generation cosmic ray experiment by updated emulsion techniques is progressing. Gamma-Ray Astro-Imager with Nuclear Emulsion (GRAINE), which is a cosmic gammaray observation project, employs a balloon-borne emulsion detector [ㅁ]]. The emulsion gamma-ray 
telescope has an angular resolution $\left(0.08^{\circ} @ 1-2 \mathrm{GeV}\right)$ that is one order of magnitude higher than that of the Fermi-LAT [[13]]. In addition, It has the polarization sensitivity using the pair creation mode. The emulsion telescope consists of the converter, which detects e-pair events and reconstructs incident angles of gamma-rays by measuring the beginning of each track angle; the time stamper, which is based on an emulsion multi-stage shifter technique [44]][प5]; and the calorimeter, which measures momentum of tracks via the multiple coulomb scattering (MCS) method [प]].

We performed the first mass production of highly sensitive emulsion films in order to introduce them to the GRAINE 2015 balloon experiment in Alice Springs, Australia. GRAINE 2015 aims to demonstrate the performance, especially the angular resolution, of the emulsion telescope by detecting the Vela pulsar. The telescope's aperture area and total area of mounted films are 3600

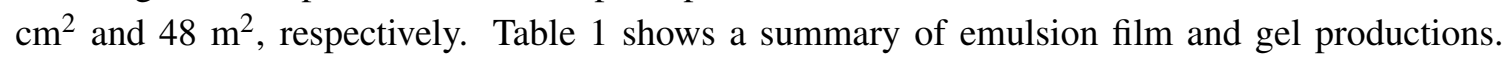
Highly sensitive films were employed for the converter in order to increase conversion probability. Moderately sensitive films were employed for the time stamper and the calorimeter in order to reduce MCS in the emulsion layer and reduce costs while retaining adequate efficiency. A total of $195 \mathrm{~kg}$ gel was produced over the period of a year. The pouring process for $67 \mathrm{~m}^{2}$ in total required about three months. Then, all films were transported to Australia and stacked in chambers.

The balloon experiment was performed on the 12th of May in 2015. The observation, recovery, and development were completed safely (the details are described in [[17]][18]][प्प]). Readout of these flight films by the HTS has been progressing since July.

Table 1: Summary of emulsion gel/film productions for GRAINE 2015 experiment

\begin{tabular}{lrrr} 
& Produced gel $^{* 1}$ & Poured gel (film) ${ }^{* 2}$ & Mounted film \\
\hline \hline Highly sensitive type & $166.4 \mathrm{~kg}$ & $122.4 \mathrm{~kg}\left(54.4 \mathrm{~m}^{2}\right)$ & $38.9 \mathrm{~m}^{2}$ \\
Moderately sensitive type & $28.2 \mathrm{~kg}$ & $21.5 \mathrm{~kg}\left(12.5 \mathrm{~m}^{2}\right)$ & $9.2 \mathrm{~m}^{2}$ \\
\hline Total & $194.7 \mathrm{~kg}$ & $143.9 \mathrm{~kg}\left(66.8 \mathrm{~m}^{2}\right)$ & $48.1 \mathrm{~m}^{2}$ \\
${ }^{* 1}$ Jan. 2014-Jun. 2014, Oct. 2014-Feb. 2015 & & \\
${ }^{* 2}$ May 2014-Jun. 2014, Dec. 2014-Mar. 2015 & \\
${ }^{* 3}$ May 2015
\end{tabular}

\section{Conclusion}

We have introduced the system of nuclear emulsion gel production to the laboratory, and have started self-development and supply of a new gel, instead of relying on the photographic film companies. Highly sensitive emulsion film, which was developed by increasing the $\mathrm{AgBr}$ content in the gelatin, realizes excellent efficiency and signal-to-noise ratio. In addition, cosmic ray experiments using an emulsion detector that features are high spatial resolution and large area are proposed together with development of the high-speed readout system (HTS): a cosmic gamma ray observation project, GRAINE; cosmic ray radiography project; and so on.

\section{Acknowledgements}

We thank Dr. T. Nakadaira, Dr. A. Ariga, and other T2K experiment members for their 
support during the muon beam exposure. We also thank Dr. K. Hoshino and Dr. K. Nakagawa for the provision of information about the emulsion pouring technique. This work was supported by JSPS KAKENHI Grant Number 26247039, 26105510 ,and Grant-in-Aid for JSPS Fellows.

\section{References}

[1] G. P. F. Occhialini and C. F. Powell., Nature, 159.1 (1947): 86-190

[2] K. Niu, et al., Progress of Theoretical Physics 46 (1971): 1644-1646.

[3] K. Hoshino et al., Proc. Int. Cosmic Ray Symp. on High Energy Phenomena, (1974) p. 149.

[4] S. Aoki, et al., Nucl. Instr. and Meth. B 51 (1990) 466

[5] K. Morishima and T. Nakano, Journal of Instrumentation 5 (2010) P04011.

[6] N. Armenise, et al. Nucl. Instr. and Meth. A 551.2 (2005): 261-270.

[7] DONUT Collaboration, Phys.Lett. B 504 (2001): 218.

[8] S. Aoki, et al., Prog. Theor. Phys. 85 (1991): 1287-1298.

[9] N. Agafonova, et al., Prog. Theor. Exp. Phys. (2014) 101C01

[10] N. Naganawa and K. Kuwabara, Abstracts for SPIJ ' s Fall Conference in 2010, pp. 10-11 [in Japanese].

[11] K. Matsuoka, et al., Nucl. Instr. and Meth. A 624.3 (2010) 591-600.

[12] S. Takahashi, et al., Prog. Theor. Exp. Phys. (2015) 043H01

[13] W. B. Atwood, et al., ApJ 697 (2009) 1071.

[14] S. Takahashi,et al., Nucl. Instr. and Meth. A 620 (2010) 192.

[15] H. Rokujo, et al., Nucl. Instr. and Meth. A 701 (2013) 127.

[16] N. Agafonova, et al., New Journal of Physics 14.1 (2012): 013026.

[17] S. Takahashi, et al., PoS(ICRC2015)970

[18] K. Ozaki, et al., PoS(ICRC2015)1004

[19] H. Rokujo, et al., PoS(ICRC2015)1021 